\title{
Parameter-Robust Linear Quadratic Gaussian Technique for Multi-Agent Slung Load Transportation
}

\author{
Hae-In Lee ${ }^{\mathrm{a}}$, Dong-Wan Yoo ${ }^{\mathrm{b}}$, Byung-Yoon Lee ${ }^{\mathrm{c}}$, Gun-Hee Moon ${ }^{\mathrm{c}}$, Dong-Yeon Lee ${ }^{\mathrm{c}}$, \\ Min-Jea Tahk ${ }^{\mathrm{c}, *}$, Hyo-Sang Shin ${ }^{\mathrm{a}}$ \\ ${ }^{a}$ Cranfield University, College Road, Cranfield, Bedfordshire, MK43 OAL, UK \\ ${ }^{b}$ The Agency of Defence Development, Yuseong, Daejeon, Korea \\ ${ }^{c}$ KAIST, 291 Daehak-ro, Daejeon, 305-701, Korea
}

\begin{abstract}
This paper copes with parameter-robust controller design for transportation system by multiple unmanned aerial vehicles. The transportation is designed in the form of string connection. Minimal state-space realization of slung-load dynamics is obtained by Newtonian approach with spherical coordinates. Linear quadratic Gaussian / loop transfer recovery (LQG/LTR) is implemented to control the position and attitude of all the vehicles and payloads. The controllers robustness against variation of payload mass is improved using parameter-robust linear quadratic Gaussian (PRLQG) method. Numerical simulations are conducted with several transportation cases. The result verifies that LQG/LTR shows fast performance while PRLQG has its strong point in robustness against system variation.
\end{abstract}

Keywords: Unmanned Aerial Vehicle, Slung-Load Dynamics, Linear Quadratic Gaussian, Loop Transfer Recovery, Parameter-Robust Control

\section{Introduction}

Recently, unmanned aerial vehicles (UAVs) are getting attention for both military and civilian uses. In the future, transportation using UAVs is also expected to be common, but a small light UAV generally does not have enough power to lift a heavy load. Rather than employing a larger UAV, cooperation of multiple UAVs can be an efficient approach for transporting various types of payload. Interconnection of multiple UAVs results in complicated equations of motion, as each UAV heavily affects the motion of the others. Importance and possibility of employing multiple UAVs in transportation has been mentioned in other previous studies [1]. Although there are many possible ways of cooperation, such as rigid gripping with clamps [2] and bar joint, string connection is chosen in this study to maintain the degree of freedom of each UAV as shown in Fig. 1.

\footnotetext{
* Corresponding Author

Email addresses: haein.lee@cranfield.ac.uk (Hae-In Lee), dwyoo@fdcl.kaist.ac.kr (Dong-Wan Yoo), bylee@fdcl.kaist.ac.kr (Byung-Yoon Lee), ghmoon@fdcl.kaist.ac.kr (Gun-Hee Moon), dylee@fdcl.kaist.ac.kr (Dong-Yeon Lee), mjtahk317@gmail.com (Min-Jea Tahk), h.shin@cranfield.ac.uk (Hyo-Sang Shin) 


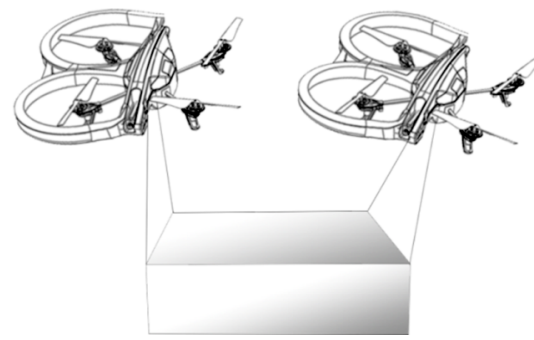

Figure 1: Transportation system with string connections

In the previous studies, a single aircraft lifting one payload with a long string has been considered [3,4]. As these cases assume a sufficiently long pendulum, coupling effects or aerodynamic disturbances on payload are negligible, and thus whole system does not have to be included in the model. To consider coupling effects into model, Maza et al. solved this problem using Kane method [5, 6, 7]. On the other hand, Bisgaard et al. [8, 9] employed Udwadia-Kalaba Equation (UKE), which is more efficient in expressing constrained dynamics. Existing modeling techniques, both Kane and UKE method, give the precise model of slung load transportation system, while state-space representations are not minimal. As one string connection reduces one degree of freedom, the minimum number of states is reduced by the number of strings. The existence of superficial states leads to a system model absent of controllability. Since the number of system variables is large and the model is complicated, model reduction is not easy. Our previous work [10] using UKE method, therefore, was not able to apply LQ-based controllers. To circumvent the controllability problem, it is suggested to utilize the combination of spherical coordinates and Cartesian coordinates [11]. The equation of motion is derived by Newtonian approach as it is easier to generalize the equation of motion compared to the Lagrangian approach. Unlike previous methods, tensile forces are computed by matrix inversion with inclusion of internal force into the state vector.

Stability analysis and control of the modeled system is another major issue in this paper. Two approaches are possible for control design: control of each UAV with respect to external disturbance including the effect of tension and control design considering the whole system. Previously, studies in [3, 4] utilize the former and Michael et al. [12, 13] perform only stability analysis. In order to conduct aggressive control in response to pendulum motion, whole system states are required to be controlled at the same time. This paper implements classical optimal control technique, linear quadratic Gaussian (LQG) technique [14]. LQG is useful to find gains for complicated transportation systems, while PID control, the most commonly used method, is hard to be implemented for large number of states. The tuning of PID gains is generally performed by trial-and-error and coupling between the longitudinal and lateral dynamics makes this tuning hard.

For practical use, it would be better to transport the payloads with various weights without changing the controller. Also, continuous loss of weight during transportation is common in agricultural uses. To improve the robustness of the LQG controller, loop transfer recovery (LTR) [15, 16] or parameter-robust linear quadratic Gaussian (PRLQG) [17, 18] can be employed. PRLQG is expected to provide better robustness then LQG/LTR. In addition to our previous work [11], frequency-domain analysis on 
stability proves the improvements in robustness.

This paper is composed as follows. First, the mathematical modeling procedure of multi-UAV slung load transportation system using Newtonian approach is presented. Second, control design theory of LQG/LTR and PRLQG method is briefly reviewed, and the transportation system model is reformulated into a moderate form for controller design process. Third, numerical simulation using MATLAB is conducted to analyze the performance of LQG/LTR and PRLQG controller. Finally, conclusion is drawn from numerical results and future work is suggested.

\section{Slung Load Transportation System Modeling}

The following sub-sections suggest modeling procedure for transportation system with Newtonian approach, assuming no aerodynamic force or fluctuation in strings. Only gravitational force and lift force of UAVs are assumed to be significant in the model. The equation of motion is generalized with unspecified number and shape of UAVs. General equation of motion is then reduced to two cases: one point mass transportation system with one UAV, and one box payload transportation with four UAVs.

\subsection{Derivation of General Equation of Motion}

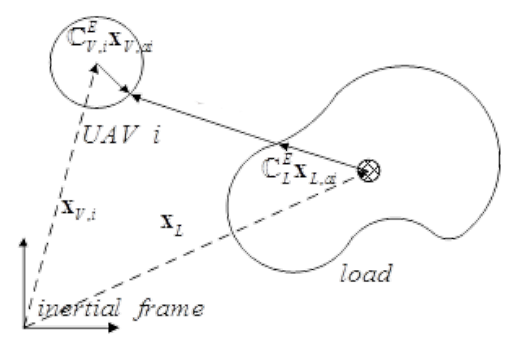

Figure 2: Transportation system nomenclature

System variables of transportation system with unspecified number of UAVs and type of payloads are shown in Fig. 2. System variables chosen for modeling are position and attitude of the load $\left(\mathbf{x}_{L}, \boldsymbol{\theta}_{L}\right)$, spherical coordinate angle of the strings $\left(\boldsymbol{\theta}_{i}\right)$, and attitude of each $\operatorname{UAV}\left(\boldsymbol{\theta}_{V, i}\right)$, where attitude information is required for computing direction cosine matrices $\left(\mathbb{C}_{V, i}^{E}, \mathbb{C}_{L}^{E}\right)$ and spherical coordinate $(C)$ is used to describe the motion of strings for constrained length. The spherical coordinate is determined so that zero angles yield hovering condition as follows:

$$
C(\boldsymbol{\theta})=l\left[\begin{array}{c}
\sin \phi \\
-\sin \theta \cos \phi \\
-\cos \theta \cos \phi
\end{array}\right]
$$

Observing the geometric relationship in Fig. 2, position states of the UAVs $\left(\mathbf{x}_{V, i}\right)$ are determined as follows. 


$$
\mathbf{x}_{V, i}=\mathbf{x}_{L}+\mathbb{C}_{L}^{E} \mathbf{x}_{L, a i}+C\left(\boldsymbol{\theta}_{i}\right)-\mathbb{C}_{V, i}^{E} \mathbf{x}_{V, a i}
$$

where $\mathbf{x}_{v, a i}$ and $\mathbf{x}_{L, a i}$ stands for the vectors from the center of mass of vehicle or load respectively to the attachment point of i-th string.

Applying Newton's 2nd law of motion and Euler equation, the following equation is the basic idea of modeling.

$$
\begin{cases}\mathbf{M}_{V, i} \ddot{\mathbf{x}}_{V, i} & =\mathbf{F}_{V, i}-\sum T_{i} C\left(\boldsymbol{\theta}_{i}\right) / l_{i} \\ \mathbf{M}_{L} \ddot{\mathbf{x}}_{L} & =\mathbf{F}_{L}+\sum T_{i} C\left(\boldsymbol{\theta}_{i}\right) / l_{i} \\ \mathbf{I}_{V} \dot{\boldsymbol{\omega}}_{V, i} & =\boldsymbol{\tau}_{V, i}-\sum T_{i}\left(\mathbf{x}_{V, a i} \times \mathbb{C}_{E}^{V, i} C\left(\boldsymbol{\theta}_{i}\right)\right) / l_{i} \\ \mathbf{I}_{L} \dot{\boldsymbol{\omega}}_{L} & =\sum T_{i}\left(\mathbf{x}_{L, a i} \times \mathbb{C}_{E}^{L} C\left(\boldsymbol{\theta}_{i}\right)\right) / l_{i}\end{cases}
$$

where $\mathbf{M}_{L}$ or $\mathbf{M}_{V, i}$ is a mass matrix with diagonal entries of mass $m_{L}$ or $m_{V, i}$, $\mathbf{I}$ is an inertial matrix, $T$ is a tensile force, $l$ is the length of a string, and $\boldsymbol{\omega}$ is the angular velocity in the body frame. The forces $\mathbf{F}_{V, i}$ and $\mathbf{F}_{L}$ include the gravitational force as

$$
\mathbf{F}_{V, i}=\mathbb{C}_{V, i}^{E} \mathbf{F}_{M, i}+\mathbf{M}_{V, i} \mathbf{g}, \quad \mathbf{F}_{L}=\mathbf{M}_{L} \mathbf{g}, \quad \mathbf{g}=[0,0, g]^{T}
$$

The inputs of the system are given as external forces $\left(\mathbf{F}_{M, i}\right)$ and moments $\left(\boldsymbol{\tau}_{V, i}\right)$ regardless of UAV dynamics. This modeling is for general type and dynamics of UAV, and thus individual dynamics must be augmented separately.

Substitution of equation (2) into equation (3) yields relationship among system variables and tensile forces. Although values of tensile forces are not measured, secondderivative terms and tensile forces have linear relationship, and thus derivative terms can be computed as an inverse matrix form as follows.

$$
\begin{aligned}
& {\left[\begin{array}{ccccc}
\ddot{\mathbf{x}}_{L}^{T} & \ddot{\boldsymbol{\theta}}_{i}^{T} & \dot{\boldsymbol{\omega}}_{L}^{T} & \dot{\boldsymbol{\omega}}_{V, i}^{T} & T_{i} / l_{i}
\end{array}\right]^{T}=} \\
& {\left[\begin{array}{ccccc}
\mathbf{M}_{V, i} & \mathbf{M}_{V, i} C^{\prime}\left(\boldsymbol{\theta}_{i}\right) & \mathbf{M}_{V, i} \mathbb{C}_{L}^{\prime E} \mathbf{x}_{L, a i} & -\mathbf{M}_{V, i} \mathbb{C}_{V, i}^{\prime E} \mathbf{x}_{V, a i} & C\left(\boldsymbol{\theta}_{i}\right) \\
\mathbf{M}_{L} & \mathbf{0}_{3 \times 2} & \mathbf{0}_{3 \times 3} & \mathbf{0}_{3 \times 3} & -C\left(\boldsymbol{\theta}_{i}\right) \\
\mathbf{0}_{3 \times 3} & \mathbf{0}_{3 \times 2} & \mathbf{0}_{3 \times 3} & \mathbf{I}_{V, i} & \mathbf{x}_{V, a i} \times \mathbb{C}_{E}^{V, i} C\left(\boldsymbol{\theta}_{i}\right) \\
\mathbf{0}_{3 \times 3} & \mathbf{0}_{3 \times 2} & \mathbf{I}_{L} & \mathbf{0}_{3 \times 3} & -\mathbf{x}_{L, a i} \times \mathbb{C}_{E}^{L} C\left(\boldsymbol{\theta}_{i}\right)
\end{array}\right]} \\
& {\left[\begin{array}{c}
\mathbf{F}_{V, i}-\sum_{i} \mathbf{M}_{V, i}\left(G\left(\boldsymbol{\theta}_{i}, \dot{\boldsymbol{\theta}}_{i}\right)+\mathbb{G}_{L}^{E} \mathbf{x}_{L, a i}-\mathbb{G}_{V, i}^{E} \mathbf{x}_{V, a i}\right) \\
\\
\mathbf{F}_{L} \\
\boldsymbol{\tau}_{V, i} \\
\mathbf{0}_{3 \times 1}
\end{array}\right]}
\end{aligned}
$$

The prime mark notes for differentiation not along the time but along the spherical coordinate states of strings. To be specific, function $C^{\prime}$ and matrix $\mathbb{C}^{\prime}$ is computed as

$$
C^{\prime} \triangleq\left[\begin{array}{ll}
\frac{d C}{d \theta} & \frac{d C}{d \phi}
\end{array}\right], \quad \mathbb{C}^{\prime} \mathbf{x}_{a i} \triangleq\left[\begin{array}{lll}
\frac{d \mathbb{C}}{d \phi} \mathbf{x}_{a i} & \frac{d \mathbb{C}}{d \theta} \mathbf{x}_{a i} & \frac{d \mathbb{C}}{d \psi} \mathbf{x}_{a i}
\end{array}\right]
$$


where the differentiated result is similar to the gradient in that the required form is an augmented matrix.

Function $G$ and matrix is introduced to cope with first-derivative terms. For instance, function $G$, which is related to spherical coordinate transformation function $C$, is defined as

$$
\begin{aligned}
G(\boldsymbol{\theta}, \dot{\boldsymbol{\theta}}) & =\frac{d^{2} C(\boldsymbol{\theta})}{d t^{2}}-C^{\prime}(\boldsymbol{\theta}) \ddot{\boldsymbol{\theta}}^{T} \\
& =-\left[\begin{array}{c}
\dot{\phi}^{2} \sin \phi \\
-\left(\dot{\theta}^{2}+\dot{\phi}^{2}\right) \sin \theta \cos \phi \\
-\left(\dot{\theta}^{2}+\dot{\phi}^{2}\right) \cos \theta \cos \phi
\end{array}\right]+2 \dot{\theta} \dot{\phi}\left[\begin{array}{c}
0 \\
\cos \theta \sin \phi \\
-\sin \theta \sin \phi
\end{array}\right]
\end{aligned}
$$

while $\mathbb{G}$ is computed similarly.

The size of the inverse matrix in equation (5) is thus $3 n+3$ for two-dimensional case and $6 n+6$ for three-dimensional case, when $n$ stands for the number of UAVs. It is the same as the summation of degree of freedom for $n+1$ agent, whereas the number of system variables reduces by $n$ as tensile forces do not belong to system variable. The number of reduced variables can be also interpreted as $n$ string constraints.

\subsection{Equation of Motion for Sample Cases}

The simplest transportation system is given with one UAV and a point mass load attached to the center of UAV with a single string. General equation of motion given in equation (5) is reduced as follows:

$$
\begin{aligned}
& {\left[\begin{array}{lll}
\ddot{\mathbf{x}}_{L}^{T} & \ddot{\boldsymbol{\theta}}^{T} & T / l
\end{array}\right]^{T}=} \\
& {\left[\begin{array}{ccc}
\mathbf{M}_{V} & \mathbf{M}_{V} C^{\prime}(\boldsymbol{\theta}) & C(\boldsymbol{\theta}) \\
\mathbf{M}_{L} & \mathbf{0}_{3 \times 2} & -C(\boldsymbol{\theta})
\end{array}\right]^{-1}\left(\left[\begin{array}{c}
\mathbf{F}_{V} \\
\mathbf{F}_{L}
\end{array}\right]-\left[\begin{array}{c}
\mathbf{M}_{V} G(\boldsymbol{\theta}, \dot{\boldsymbol{\theta}}) \\
\mathbf{0}_{3 \times 1}
\end{array}\right]\right)}
\end{aligned}
$$

As the string is connected to the center of UAV, attitude of both load and UAV does not influence the whole system behavior. However, in case of a quadrotor-type UAV, pitch and roll attitude can be determined by the relationship among external force vectors, as it generates thrust mainly in $z$-axis direction.

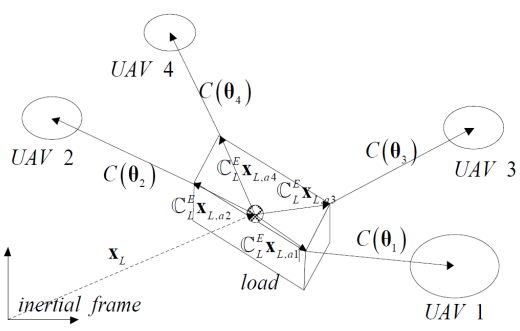

Figure 3: Transportation system with four UAVs and a box payload

Expanding the modeling into four UAVs and a box, variables are determined as in Fig. 3. When setting connection points to the payload, one must be careful not to create uncontrollable mode. For instance, if all the strings are attached to a single point or 

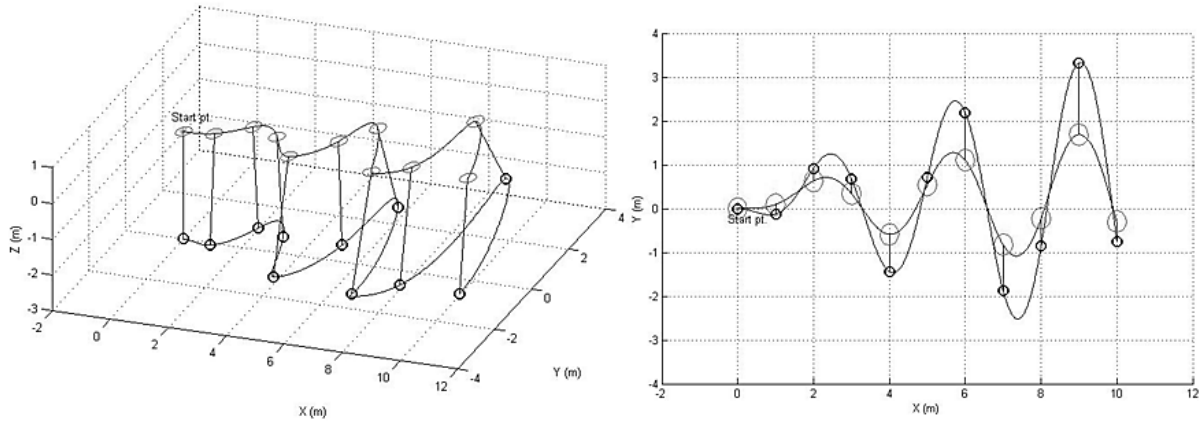

Figure 4: Verification of slung load dynamics excited with natural frequency

divided to two points, the matrix in the equation (5) becomes singular and at least one mode of payload is not controllable. In this paper, four UAVs are connected to the upper points of the box as shown in Fig. 3. Even though the attachment points are determined, $\mathbf{x}_{L, a i} \mathrm{~S}$ differ by the size of load. The resultant equation of motion is

$$
\begin{aligned}
& {\left[\begin{array}{cccccc}
\ddot{\mathbf{x}}_{L}^{T} & \ddot{\boldsymbol{\theta}}_{1}^{T} & \cdots & \dot{\boldsymbol{\omega}}_{L}^{T} & T_{1} / l_{1} & \cdots
\end{array}\right]^{T}=} \\
& {\left[\begin{array}{cccccc}
\mathbf{M}_{V, 1} & \mathbf{M}_{V, 1} C^{\prime}\left(\boldsymbol{\theta}_{1}\right) & \mathbf{0}_{3 \times 2(n-1)} & \mathbf{M}_{V, 1} \mathbb{C}_{L}^{\prime} \mathbf{x}_{L, a 1} & C\left(\boldsymbol{\theta}_{1}\right) & \mathbf{0}_{3 \times(n-1)} \\
\vdots & \mathbf{0}_{3(n-1) \times 2} & \ddots & \vdots & \mathbf{0}_{3(n-1) \times 1} & \ddots \\
\mathbf{M}_{L} & \mathbf{0}_{3 \times 2} & \mathbf{0}_{3 \times 2(n-1)} & \mathbf{0}_{3 \times 3} & -C\left(\boldsymbol{\theta}_{1}\right) & \cdots \\
\mathbf{0}_{3 \times 3} & \mathbf{0}_{3 \times 2} & \mathbf{0}_{3 \times 2(n-1)} & I_{L} & \mathbf{x}_{L, a 1} \times \mathbb{C}_{E}^{L} C\left(\boldsymbol{\theta}_{1}\right) & \cdots
\end{array}\right]} \\
& {\left[\begin{array}{c}
\mathbf{F}_{V, 1}-\sum_{i} \mathbf{M}_{V, i}\left(G\left(\boldsymbol{\theta}_{i}, \dot{\boldsymbol{\theta}}_{i}\right)+\mathbb{G}_{L}^{E} \mathbf{x}_{L, a i}\right) \\
\vdots \\
\\
\\
\\
\quad \mathbf{F}_{L} \\
\mathbf{0}_{3 \times 1}
\end{array}\right]}
\end{aligned}
$$

\subsection{Verification with Udwadia-Kalaba Equation}

To verify the modeling, comparison with UKE is suggested [9-10]. Among all the previous works, modeling using UKE considered the most general case when the strings are attached to the different point with center of mass, and thus appropriate to be compared with the model suggested in this thesis. UKE enables explicit computation of internal forces and moments with unconstrained states $\left(\mathbf{q}_{u}\right)$, which is simply computed as

$$
\ddot{\mathbf{q}}_{u}=\mathbf{M}^{-1}\left[\begin{array}{llll}
\mathbf{F}_{V, i}^{T} & \boldsymbol{\tau}_{V, i}^{T} & \mathbf{F}_{L}^{T} & \mathbf{0}_{1 \times 3}
\end{array}\right]^{T}
$$

where augmented mass matrix $\mathbf{M}$ consists of mass of all the states, and the states are composed of positions and attitudes of UAVs and payload. Utilizing the unconstrained 
states, constraint force and moments are computed as

$$
F_{c}=\mathbf{M}^{\frac{1}{2}}\left(A_{1} \mathbf{M}^{-\frac{1}{2}}\right)^{+}\left(A_{2}-A_{1} \ddot{\mathbf{q}}_{u}\right)
$$

where $F_{C}$ is different from tensile force as it includes forces and moments for all the state variables. Matrices $A_{1}$ and $A_{2}$ are defined in order to present string constraints as

$$
\begin{aligned}
& A_{1}=L_{i}^{T}\left[\begin{array}{llll}
\mathbb{C}_{V, i}^{E} & -\mathbb{C}_{V, i}^{E} \tilde{\mathbf{x}}_{V, a i} & -\mathbb{C}_{L}^{E} & \mathbb{C}_{L}^{E} \tilde{\mathbf{x}}_{L, a i}
\end{array}\right] \\
& A_{2}=\dot{L}_{i}^{T} \dot{L}_{i} \\
& -L_{i}^{T}\left(\mathbb{C}_{V, i}^{E} \tilde{\omega}_{V, i}^{2} \mathbf{x}_{V, a i}+\mathbb{C}_{V, i}^{E} \tilde{\mathbf{x}}_{V, a i} I_{V}^{-1} \tilde{\omega}_{V, i} I_{V} \omega_{V, i}\right. \\
& \left.-\mathbb{C}_{L}^{E} \tilde{\omega}_{L}^{2} \mathbf{x}_{L, a i}-\mathbb{C}_{L}^{E} \tilde{\mathbf{x}}_{L, a i} I_{L}^{-1} \tilde{\omega}_{L} I_{L} \omega_{L}\right)
\end{aligned}
$$

where $L$ is a vector notation of strings as same as in Fig. 2. Using the equations from (12) to (13), result of equation (11) yields constrained states as follows.

$$
\ddot{\mathbf{q}}_{b}=\ddot{\mathbf{q}}_{u}+\mathbf{M}^{-1} F_{c}
$$

The UKE approach can be also represented by Newtonian approach similarly. Defining all the states the same, equation (11) and (14) can be unified in the form of equation (5) as

$$
\left[\begin{array}{c}
\ddot{\mathbf{q}}_{b} \\
T
\end{array}\right]=\left[\begin{array}{cc}
\mathbf{M} & A_{1}^{T} \\
A_{1} & 0
\end{array}\right]^{-1}\left[\begin{array}{c}
\mathbf{F}_{V, i} \\
\boldsymbol{\tau}_{V, i} \\
\mathbf{F}_{L} \\
\mathbf{0}_{3 \times 1} \\
0
\end{array}\right]
$$

In order to verify the modeling, simple case when one UAV is lifting one point mass payload is considered numerically. As two modeling methods have different choices of system states, spherical coordinates of Newtonian approach are converted to Cartesian coordinates for reference. The resultant second derivatives showed error less than the order of truncation error, and thus it can be concluded that the two modeling methods are correct. Also, when the lift is given with sinusoidal form in natural frequency $\sqrt{g / l}$, the pendulum motion enlarges as shown in Fig. 4. As swinging angle of the payload increases, coupling force on UAV enlarges the trajectory of UAV. Intuitively, it can be concluded that the moving pendulum dynamics and coupling effect is well-modeled.

\section{Control Design of Multi-UAV Transportation System}

This section outlines design of controller. Classic control method LQG is implemented in more analytic perspective. Then, PRLQG is added in order to improve robustness along parameter variation. Finally, the modeled systems in section 2 are modified in an appropriate form for control techniques. 


\subsection{Linear Quadratic Gaussian / Loop Transfer Recovery Technique}

LQG is to optimize the cost of state errors and input in linearized state-space form. Even though not all the states are observed, estimator is designed to compensate the error. Formulation of controller and estimator is presented as follows.

$$
\begin{aligned}
& \dot{x}=A x+B u, \quad y=C x \\
& u=-K_{C} \hat{x}, \quad \dot{\hat{x}}=\left(A-K_{F} C\right) \hat{x}+B u+K_{F} y
\end{aligned}
$$

Through optimization method, gains are obtained by the solution of the following algebraic Riccati equation (ARE).

$$
\begin{aligned}
& K_{C}=R^{-1} B^{T} P_{C}, P_{C} A+A^{T} P_{C}+Q-P_{C} B R^{-1} B^{T} P_{C}=0 \\
& K_{F}=P_{F} C^{T} W^{-1}, P_{F} A^{T}+A P_{F}+V-P_{F} C^{T} W^{-1} C P_{F}=0
\end{aligned}
$$

Design parameters $Q, R, V$ and $W$ are determined by relative weighting among states, state and command, model accuracy, and sensor accuracy, respectively. As these design parameters have patterns, expanding the transportation system does not influence hardly on determining them, and it acts as a strong advantage compared to PID control technique.

As LQG may not have desirable robustness, LTR is augmented to recover open-loop gain. LTR is conducted by modifying the design parameters to get sensitivity recovery or robustness recovery as follows.

$$
\begin{aligned}
& \text { robustness recovery: } \quad Q=Q_{0}+w_{C, L T R}^{2} C^{T} C \\
& \text { sensitivity recovery: } \quad V=V_{0}+w_{F, L T R}^{2} B B^{T}
\end{aligned}
$$

While increasing the weight of LTR $\left(w_{L T R}^{2}\right)$ improves robustness or sensitivity, the controller gains also increase along with the weight of LTR, resulting in the aggressive maneuvre. Considering both robustness and performance, $w_{L T R}^{2}$, is selected to have the open-loop gain as close as possible to the target loop function at crossover frequency. The target loop function is defined as a transfer function to which the open-loop gain theoretically converges as $w_{L T R}^{2}$ approaches infinity. The LQG/LTR open-loop gain $G_{2}$ and the target loop function $G_{1}$ are computed by following equations.

$$
\begin{aligned}
& G_{1}(s)=K_{C}(s I-A)^{-1} B \\
& G_{2}(s)=K_{C}\left(s I-A+B K_{C}+K_{F} C\right)^{-1} K_{F} C(s I-A)^{-1} B
\end{aligned}
$$

Two important assumptions for applying LTR are that the system must be minimum phase and the number of measured states needs to be larger than that of control inputs. If the system has right half-plane closed-loop poles, the extra phase lead contributed by these poles is required for maintaining stability. Also, measurements should be sufficient for adding dummy columns to $B$ and zero rows to $K_{C}$ to make $C(s I-A)^{-1} B$ and $K_{C}(s I-A)^{-1} B$ square matrices. As $C(s I-A)^{-1} B$ must remain as a minimum phase system, enlarging $C$ matrix or using smaller number of measurements is inappropriate.

For simple transportation system with one UAV lifting a point mass payload, actual and target loop functions from equation (19) near the crossover frequency are given in Fig. 5. Weightings are given for robustness recovery, and transfer function is from $x$-axis to $x$-axis $\left(G_{x x}\right)$ to show fluctuation control. Weighting value of 100 is expected to be 
most appropriate to make the transfer function closest to the target function. Resultant root locus is shown in Fig. 6. The system dynamics has two poles at the origin and another two poles on the imaginary axis as equation (20).

$$
G_{x x}=\frac{\omega_{n}^{2}}{m_{V} s^{2}\left(s^{2}+\frac{m_{V}+m_{L}}{m_{V}} \omega_{n}^{2}\right)}
$$

where $\omega_{n}$ is a natural frequency $\sqrt{g / l}$

Four poles and three zeros are added from LQG or LQG/LTR controller, and two zeros among them are located near to the system poles. It can be expected that the system changes might cause large change in root locus shape.

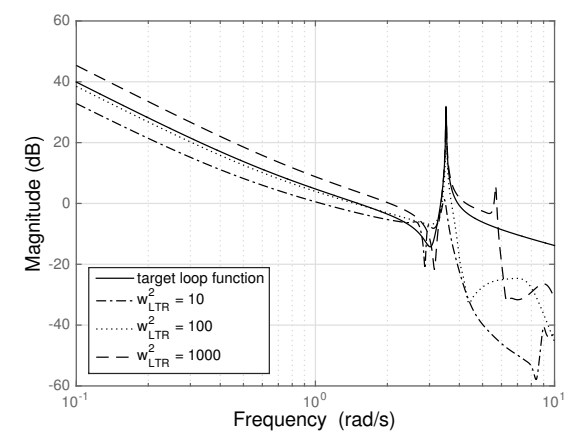

Figure 5: Bode plot of LQG/LTR with different weightings

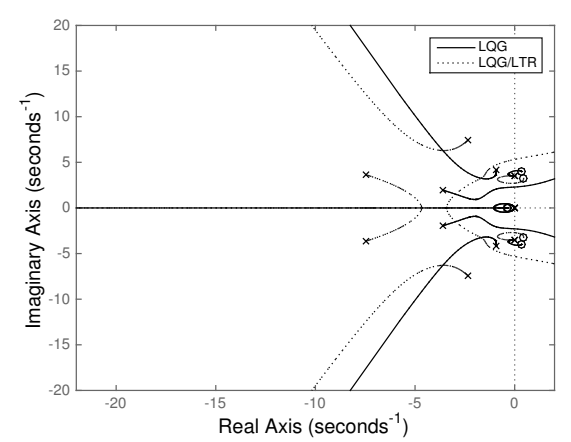

Figure 6: Root locus of LQG and LQG/LTR

\subsection{Parameter-Robust Linear Quadratic Gaussian}

PRLQG is applied to the system in which structure uncertainties such as parameter variation is expected. The estimated parameter change is reformulated in a fictitious loop called internal feedback loop (IFL), and then controller is designed to maintain 
robustness against the IFL. In order to implement PRLQG, perturbation of linearized system matrix $A$ caused by parameter variation is decomposed as follows.

$$
\Delta A=M \varepsilon N
$$

where matrix $M$ is column-similar to the system matrix $A$. Using the decomposed matrices $M$ and $N$, design parameters introduced in the previous session are modified with the following equations.

$$
\begin{aligned}
& Q=Q_{0}+w_{C, P R L Q G}^{2} N^{T} N \\
& V=V_{0}+w_{F, P R L Q G}^{2} M M^{T}
\end{aligned}
$$

where $w_{L T R}^{2}$ can still be added in case of losing controllability. The ratio between the weight of LTR and that of PRLQG is varied according to the design purpose. If robustness to the parameter variation is considered to be more significant than fast performance, the weight of PRLQG must be increased compared to that of LQG/LTR. When the mass of payload is exact enough, the weighting of PRLQG is not required to be large. Increasing the weight of PRLQG, the system's robustness function $G_{2}$ approaches to the target loop function $G_{1}$.

$$
\begin{aligned}
G_{1}(s) & =N\left(s I-A+B K_{C}\right)^{-1} M \\
G_{2}(s) & =N(s I-A)^{-1} M \\
& -N(s I-A)^{-1} B K(I+G K)^{-1} C(s I-A)^{-1} M
\end{aligned}
$$

where function $K$ and $G$ is defined as

$$
\begin{aligned}
& K(s)=K_{C}\left(s I-A-B K_{C}+K_{F} C\right)^{-1} K_{F} \\
& G(s)=C(s I-A)^{-1} B
\end{aligned}
$$

As robustness function is the transfer function from parameter variation to output, gain is required to be similar throughout the range of interest.

Similar to the previous subsection, one UAV transportation system is considered for instance. As system matrix $A$ is obtained as follows,

$$
A=\left[\begin{array}{ccccc}
0 & 0 & 0 & 0 & g \\
0 & 0 & 0 & -g & 0 \\
0 & 0 & 0 & 0 & 0 \\
0 & 0 & 0 & -\frac{m_{V}+m_{L}}{m_{V} l} g & 0 \\
0 & 0 & 0 & 0 & -\frac{m_{V}+m_{L}}{m_{V} l} g
\end{array}\right]
$$

column-similar matrices $M$ and $N$ are defined as

$$
\Delta A=M \varepsilon N=\left[\begin{array}{cc}
0 & 0 \\
0 & 0 \\
0 & 0 \\
-\frac{g}{m_{V l} l} & 0 \\
0 & -\frac{g}{m_{V} l}
\end{array}\right] \Delta m_{L}\left[\begin{array}{lllll}
0 & 0 & 0 & 1 & 0 \\
0 & 0 & 0 & 0 & 1
\end{array}\right]
$$


Bode plot of resultant transfer functions from mass variation is shown in Fig. 7. Compared with the result of LQG/LTR, PRLQG control shows relatively steady gain against parameter variations, whereas target function has more steady gain. As seen in the bode plot, the mass variation is magnified up to $42 \mathrm{~dB}$ with LQG/LTR, where the magnitude of the transfer function of PRLQG is upper bounded by $-5 \mathrm{~dB}$. Hence, less variation on performance along the parameter change is expected.

From the root locus of PRLQG in Fig. 8, zeros of the controller are placed near to the system poles at real axis. From the root locus shown in Fig. 6, it is clear that the system with LQG/LTR easily becomes non-minimum phase. On the other hand, Fig. 8 shows that parameter change does not cause large change in root locus trajectory of PRLQG. Therefore, improvements in robustness against parameter change is evident both from bode plot and root locus.

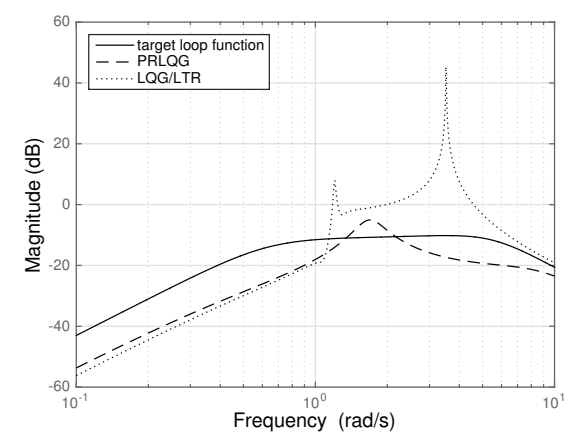

Figure 7: Bode plot of PRLQG

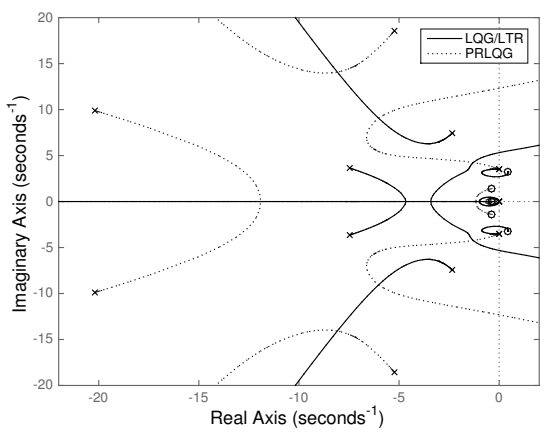

Figure 8: Root locus of PRLQG

\section{Numerical Result}

In this section, simulation setups and numerical results are presented. Specifications of UAVs and payload, and design parameters of controller introduced in previous sections 
Table 1: Slung load system specification

\begin{tabular}{cc|c|c}
\hline & & Case 1 & Case 2 \\
\hline UAV & Number & 1 & 4 \\
& Mass $(\mathrm{kg})$ & \multicolumn{2}{|c}{0.408} \\
& Size $(\mathrm{m})$ & \multicolumn{2}{|c}{$\phi 0.356$} \\
& Inertia $\left(\mathrm{kg} \cdot \mathrm{m}^{2}\right)$ & $2.2842 \cdot 10-3 \times 2.4451 \cdot 10-3 \times 4.4562 \cdot 10-3$ \\
\hline Payload & Type & Point mass & Box \\
& Mass $(\mathrm{kg})$ & 0.1 & 0.4 \\
& Size $(\mathrm{m})$ & None & $0.2 \times 1.0 \times 1.0$ \\
\hline String & Mass $(\mathrm{kg})$ & \multicolumn{2}{|c}{ None } \\
& Size $(\mathrm{m})$ & \multicolumn{2}{|c}{1.4} \\
\hline
\end{tabular}

are set. Then, result of applying the setups is presented.

\subsection{Simulation Setups}

The simulation is set with a single UAV and four-UAV transportation system. Specification of UAV and payload is given in Table 1. Size and weight of all the UAVs are set to be identical. The modelled mass of payload is set to be constant, and the mass or thickness of string is neglected. The type of UAV is selected as a small quad-rotor, AR. Drone 2.0 from Parrot, which shows the most stable hovering performance in its mass and size. Control allocation from rotational speed of rotors to force and moment of AR. Drone is defined as

$$
\begin{gathered}
\mathbf{F}_{M}=\left[\begin{array}{c}
0 \\
0 \\
-4 K_{t, 0}-K_{t}\left(\Omega_{1}^{2}+\Omega_{2}^{2}+\Omega_{3}^{2}+\Omega_{4}^{2}\right)
\end{array}\right] \\
\boldsymbol{\tau}_{V}=\left[\begin{array}{c}
K_{t}\left(-\Omega_{1}^{2}-\Omega_{2}^{2}+\Omega_{3}^{2}+\Omega_{4}^{2}\right) d+I_{r} q\left(\Omega_{1}-\Omega_{2}+\Omega_{3}-\Omega_{4}\right) \\
K_{t}\left(\Omega_{1}^{2}-\Omega_{2}^{2}-\Omega_{3}^{2}+\Omega_{4}^{2}\right) d-I_{r} p\left(\Omega_{1}-\Omega_{2}+\Omega_{3}-\Omega_{4}\right) \\
4 K_{r, 0}+K_{r}\left(\Omega_{1}^{2}-\Omega_{2}^{2}+\Omega_{3}^{2}-\Omega_{4}^{2}\right)
\end{array}\right]
\end{gathered}
$$

where $d$ is the distance from the center of UAV to rotors, $I_{r}$ is the inertia of UAV, and $p$ and $q$ are the roll and pitch rate, respectively. Also, $\Omega_{i}$ 's are the rotational speed of each rotor, saturated by maximum of $4787.1 \mathrm{rpm}$, and the rotor coefficients for thrust computation and torque are given as follows:

$$
\begin{gathered}
K_{t}=8.1763 \cdot 10^{-6}, \quad K_{t, 0}=0.0562 N \\
K_{r}=2.1703 \cdot 10^{-7}, \quad K_{r, 0}=1.0950 \cdot 10^{-4} \mathrm{~N} \cdot \mathrm{m}
\end{gathered}
$$

For the details of quad-rotor dynamics, refer to [19].

In order to evaluate the controller's robustness against the mass variation, numerical simulations are conducted with four different cases: when the actual mass (1) is equivalent 
to the modelled mass, noted as the standard case, (2) has a constant mismatch with the modelled value with $-20 \%$, (3) has again a constant difference with $+20 \%$, and (4) is varying from $+20 \%$ to $-20 \%$ with a constant rate during the transportation. Also, to analyze the effect of time delay and maximum thrust, $0.1 \mathrm{sec}$ of motor delay is applied, and maximum rotor speed is reduced by $-20 \%$. The effects are shown by the response in $x$-axis the same command $(1,1,-1) \mathrm{m}$, as the designed payload transportation system is symmetric in $x$-y axis. Compared to $z$-axis performance, $x$ - and $y$-axis response has more district changes, as perturbation of $A$ matrix in equation (26) lies in the string motion influencing mainly on $x-y$ motion of the load.

Design parameters for the LQG-based controllers are set as follows: $Q$ is 5 and 1, and $W$ is 0.1 and 1 for non-derivative terms and first derivative terms respectively. Diagonal terms of $R$ for all the states are set 10, and those of $V$ are 0.01. These rules are applied not only for the two cases we are dealing with, but also for further expansion of transportation system.

MATLAB simulation structure for one UAV and a point mass is given in Fig. 9, while larger transportation system is composed in similar sense. From dynamics, positions and attitudes of load and string is given, which is later used in calculating position of UAVs. Summation of input trim enables the linearized dynamics to be approximated to nonlinear dynamics. The command is given in feedforward structure with $\bar{N}$ matrix given as

$$
\bar{N}=N_{u}+K_{C} N_{x}, \quad \text { where }\left[\begin{array}{l}
N_{x} \\
N_{u}
\end{array}\right]=\left[\begin{array}{cc}
A & B \\
C & D
\end{array}\right]^{-1}\left[\begin{array}{l}
0 \\
1
\end{array}\right]
$$

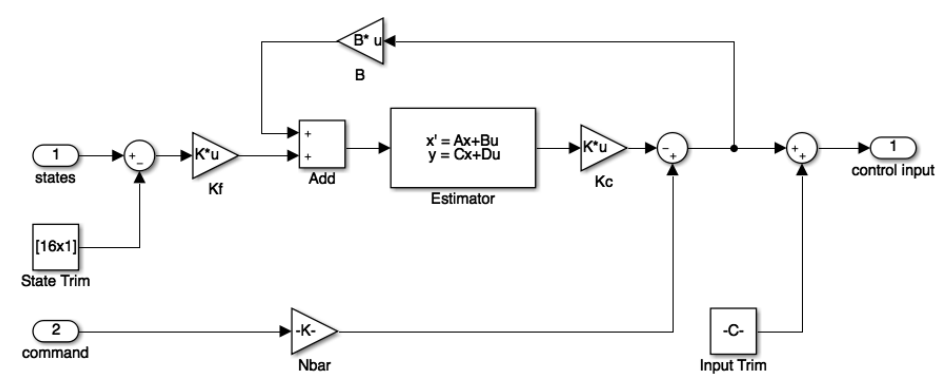

Figure 9: MATLAB Simulink Structure

\subsection{Simulation Results}

Simulation is first conducted for the single UAV slung load transportation system. The effect of variation in payload mass is demonstrated via the step responses of LQG/LTR and PRLQG in Fig. 10. While the standard response of the LQG/LTR controller is fast and fluctuating, the system controlled by PRLQG has longer settling time but stable response. When there is a difference between the actual and modelled payload mass, the fluctuation in the LQG/LTR-controlled response is increased, resulting in instability for the cases with $-20 \%$ and continuous variation. However, the responses of the PRLQGcontrolled system remain similar in all cases. It is clearly shown that the PRLQG technique improves the robustness against the payload variation as expected from Section III. 
The effect of time delay and motor thrust is shown in Fig. 11. Where the LQG/LTR controller shows unstable performance in the presence of time delay, the step response of the PRLQG control remains similar. The phase margin of the PRLQG is $69.5779 \mathrm{deg}$, whereas the phase margin of the LQG/LTR control is $11.0032 \mathrm{deg}$. The result implies that the PRLQG improves not only the robustness against parameter variation, but the phase margin. Reducing the maximum rotor speed seems to suppress the maneuvre, which means that the LQG/LTR controller without saturation has aggressive maneuvre. Increasing the PRLQG gain on the other hand smoothens the response. The problem of having higher gains of the LQG/LTR control can be resolved through applying the PRLQG.

Second, the step response of LQG/LTR and PRLQG of four-UAV slung load system is shown in Fig. 12. Similar to a single UAV system, the LQG/LTR controller shows a fluctuating, aggressive maneuvre. In the presence of mass variation, the response further fluctuates and even becomes unstable. On the other hand, the PRLQG method shows stable performance, proving the improvements in the robustness against parameter variation. Although the root locus and bode plot are not analyzed for the slung load system with more than one UAV, simulation results demonstrate enhanced robustness against the payload's mass variation with the PRLQG method.

The responses with time delay and reduction of motor thrust in four-UAV system is shown in Fig. 13. The LQG/LTR controller shows instability against the delay, implying that the phase margin is improved through the PRLQG method. Also, lower limit of saturation in the maximum thrust results in more suppression in the LQG/LTRcontrolled system, while only the overshoot is suppressed in the PRLQG method. Similar to a single UAV system, the PRLQG method does not result in aggressive maneuvre.

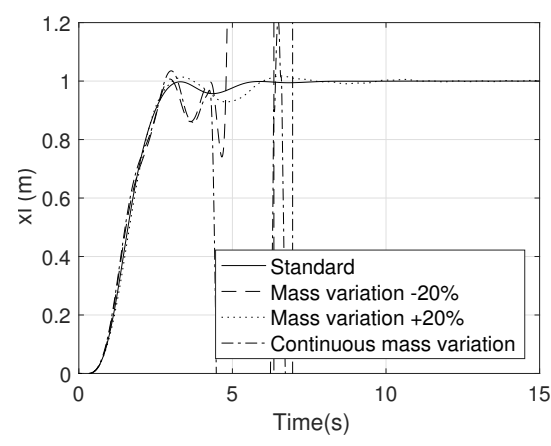

(a) LQG/LTR

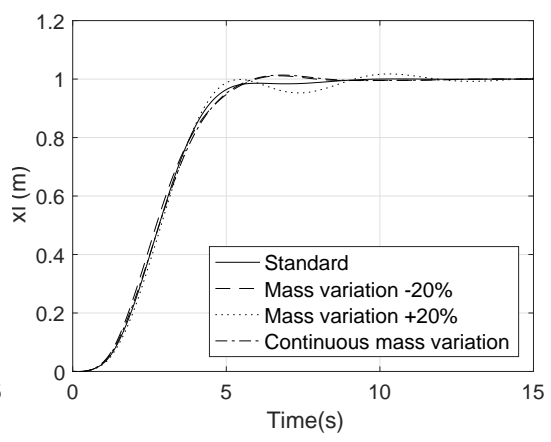

(b) PRLQG

Figure 10: Effect of payload variation in 1-UAV transportation system

\section{Conclusion}

In this paper, transportation system with multiple UAVs and string connection is modeled and controlled. Modeling is conducted with Newtonian method, combining spherical coordinates with Cartesian coordinates and using matrix inversion to compute tensile force. The proposed modeling method uses less number of states compared to 


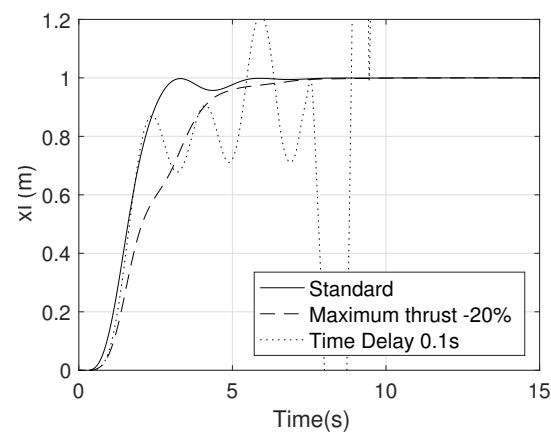

(a) LQG/LTR

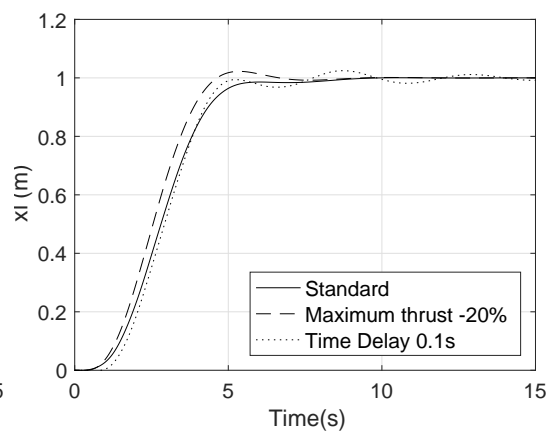

(b) PRLQG

Figure 11: Effect of system parameters in 1-UAV transportation system

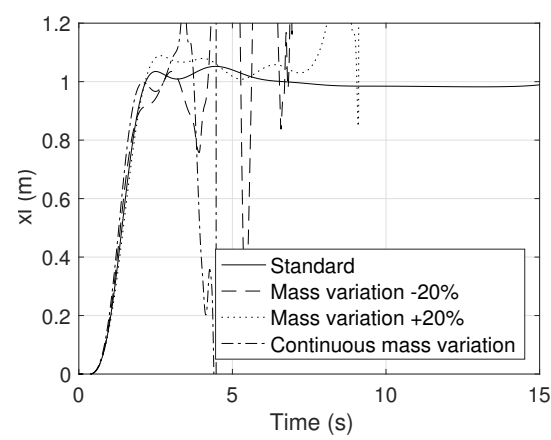

(a) LQG/LTR

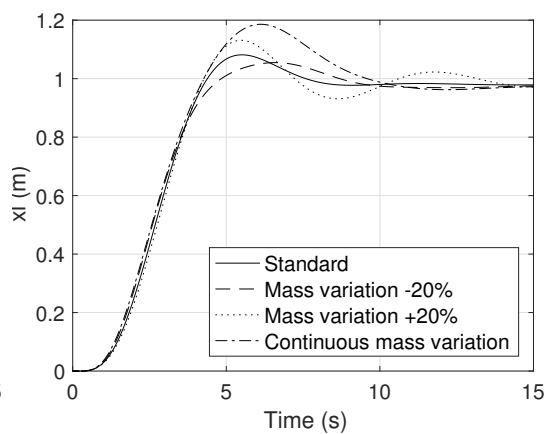

(b) PRLQG

Figure 12: Effect of payload variation in 4-UAV transportation system

other previous methods, resulting in smaller size of matrix inversion. Therefore, the proposed method is expected to reduce the computational load. Moreover, the fact that LQG gains can be obtained implies that the linearized system is controllable. The corresponding controller is designed using PRLQG technique. While previous studies did not focus on controller or utilized simple PID controllers, applying analytic control technique is a significant idea of this research. Also, implementation of the PRLQG method, which is not well-known compared to classic controls like LQG/LTR, resulted in improvements in transporting varying mass of payload. Computer simulation shows simple verification of modeling and analysis of improvements not only in parameter robustness of controller, but also in phase margin and smooth performance.

Further studies are expected to include more details in cable modeling. Slackening of the wires or even loosing tension during the transportation would be a practically significant issue. Snapping of the cables would be modelled by excluding the corresponding UAVs from the constraint model, and adding on extra tension when it is first tightened. Also, integration of unit length commonly used in architecture would be applied to model the elastricity of long wires. Consideration of aerodynamic forces would be another prac- 


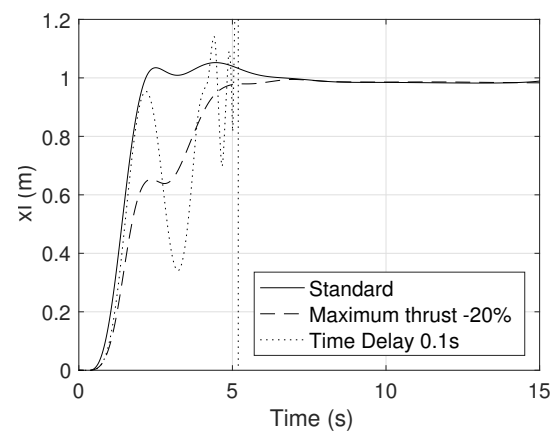

(a) LQG/LTR

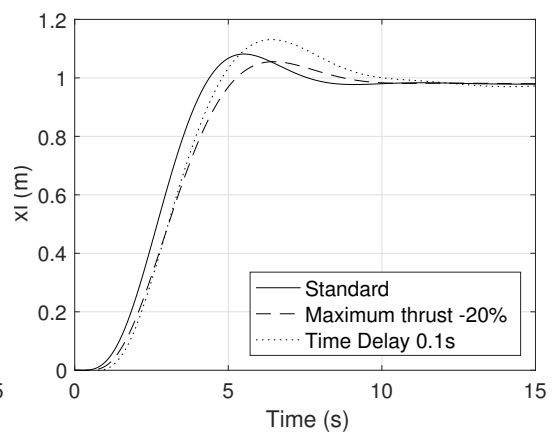

(b) PRLQG

Figure 13: Effect of system parameters in 4-UAV transportation system

tical challenge. The effect of thrust of the UAV on the payloads might require further investigation, especially in consideration of oscillation, unpredicted behaviors, and even loss of stability. This issue, which is subject to future study, could be resolved either by refining the model to include the aerodynamic forces or by designing the controller robust to the wind disturbance. Experiments would be a valuable verification for the modeling.

Another important issue is rotor failures. In system matrices, partial reduction of performance due to the rotor failures are directly related to the variation in $B$ matrix, which can be covered by PRLQG method. It is expected to improve the robustness against the failures compared with the other linear controllers. Apart from linear controllers, applying adaptive controllers would be another interesting issue, as the parameter uncertainty can be structured.

\section{Acknowledgement}

The authors would like to acknowledge support from Defense Acquisition Program Administration and Agency for Defense Development under the contract UE124026JD.

\section{References}

[1] Y. Feng, C. A. Rabbath, C.-Y. Su, Modeling of a Micro UAV with Slung Payload, in: Handbook of Unmanned Aerial Vehicles, 2015, pp. 2787-2811. doi:10.1007/978-90-481-9707-1.

URL http: //www. scopus. com/inward/record. url?eid=2-s2 .0-84944626259\&partnerID=tZDtx3y1

[2] D. Mellinger, Q. Lindsey, M. Shomin, V. Kumar, Design, modeling, estimation and control for aerial grasping and manipulation, IEEE International Conference on Intelligent Robots and Systemsdoi:10.1109/IROS.2011.6048556.

[3] R. A. Stuckey, Mathematical Modelling of Helicopter Slung-Load Systems, DSTO Aeronautical and Maritime Research Laboratory.

[4] I. Palunko, R. Fierro, P. Cruz, Trajectory Generation for Swing-Free Maneuvers of a Quadrotor with Suspended Payload : A Dynamic Programming Approach, IEEE International Conference on Robotics and Automation.

[5] M. Bernard, K. Kondak, Generic Slung Load Transportation System Using Small Size Helicopters, IEEE International Conference on Robotics and Automation. 
[6] M. Bernard, K. Kondak, I. Maza, A. Ollero, Autonomous Transportation and Deployment with, Journal of Field Robot 28 (6) (2011) 914-931.

[7] I. Maza, K. Kondak, M. Bernard, A. Ollero, Multi-UAV cooperation and control for load transportation and deployment The original publication is available at www.springerlink.com in this link :, Journal of Intelligent Robot Systems 57 (2010) 417-449.

[8] M. Bisgaard, Modeling, Estimation, and Control of Helicopter Slung Load System, Ph.D. thesis, Aalborg University (2007).

[9] M. Bisgaard, J. D. Bendtsen, A. Cour-harbo, Modeling of Generic Slung Load System, Journal of Guidance, Control, and Dynamics 32 (2) (2009) 573-585. doi:10.2514/1.36539.

[10] B. Y. Lee, H. I. Lee, D. W. Yoo, G. H. Moon, D. Y. Lee, Y. Y. Kim, M. J. Tahk, Study on payload stabilization method with the slung-load transportation system using a quad-rotor, 2015 European Control Conference, ECC 2015 (2015) 2097-2102doi:10.1109/ECC.2015.7330849.

[11] H.-I. Lee, B.-Y. Lee, D.-W. Yoo, G.-H. Moon, M.-J. Tahk, Dynamics Modeling and Robust Controller Design of the Multi-Uav Transportation System, 29th Congress of the International Council of the Aeronautical Sciences.

[12] N. Michael, S. Kim, J. Fink, V. Kumar, Kinematics and Statics of Cooperative Multi-Robot Manipulation with Cables, Proceedings of the International Design Engineering Technical Conferences 8 (2009) 83-91.

[13] N. Michael, J. Fink, V. Kumar, Cooperative manipulation and transportation with aerial robots, Autonomous Robots (September 2010) (2010) 1-14. doi:10.1007/s10514-010-9205-0.

[14] K. Morris, Introduction to Feedback Control, Harcourt/Academic Press, 2001.

[15] J. Doyle, G. Stein, Multivariable feedback design: Concepts for a classical/modern synthesis, Automatic Control, IEEE Transactions on 26 (1) (1981) 4-16. doi:10.1109/TAC.1981.1102555.

[16] G. Stein, M. Athans, The LQG / LTR Procedure for Multivariable Feedback Control Design, IEEE Transactions on Automatic Control 32 (2) (1987) 105-114.

[17] M.-J. Tahk, J. L. Speyer, Modeling of Parameter Variations and Asymptotic LQG Synthesis, IEEE Transactions on Automatic Control 32 (9) (1987) 793-801. doi:10.1109/TAC.1987.1104723.

[18] M.-J. Tahk, J. L. Speyer, Parameter Robust Linear- Quadratic-Gaussian Design Synthesis with Flexible Structure Control Applications, Journal of Guidance, Control, and Dynamics, 12 (4) (1988) 460-468.

[19] V. Ghadiok, Autonomous Aerial Manipulation Using a Quadrotor (2011). 
2017-09-14

Parameter-robust linear quadratic

Gaussian technique for multi-agent

slung load transportation

\author{
Lee, Hae-In
}

Elsevier

Lee H-I, Yoo D-W, Lee B-Y, et al., (2017) Parameter-robust linear quadratic Gaussian technique for multi-agent slung load transportation. Aerospace Science and Technology, Volume 71, December 2017, pp. 119-127

http://dx.doi.org/10.1016/j.ast.2017.09.014

Downloaded from Cranfield Library Services E-Repository 